\title{
Ultrashort laser pulse characterization techniques
}

\section{Técnicas de caracterización de pulsos laser ultracortos}

\author{
Roberto Ortega-Martínez(A) \\ Laboratorio de Pulsos Ultracortos, CCADET, UNAM, Ciudad Universitaria, Coyoacan, D.F., 04510, México. \\ (")Email: roberto.ortega@ccadet.unam.mx \\ A: miembro de AMO / AMO member \\ Recibido / Received: 07/09/2012. Revisado / Revised: 16/11/2012. Aceptado / Accepted: 04/01/2013.
}

DOI: $\underline{\text { http://dx.doi.org/10.7149/0PA.46.1.39 }}$

\begin{abstract}
:
There are several competing techniques for the characterization of ultrashort laser pulses. Here we report the studies that we have done in the laboratory. These techniques use the autocorrelation or cross-correlation of the signals. The spectral properties of almost-Gaussian functions were applied for the second-order approximation of the coefficients of Hermite-Gaussian functions that described almost perfect pulses. Other techniques are discussed such as frequency-resolved optical-gating (FROG), which is a time-dependent intensity and phase of the laser pulse, the sonogram of an ultrashort pulse using a two-photon detector which is sensitive and robust, SPIDER which measures the interference between two pulses separated in time which are identical except for their central frequencies. Finally, the ambiguity-Wigner function properties were applied for the characterization of ultrashort pulses.
\end{abstract}

Key words: Ultrashort Laser Pulses, Autocorrelation, Cross-Correlation, Hermite-Gauss Functions, FROG, SPIDER, Ambiguity Function, Wigner Function.

\section{RESUMEN:}

Existen varias técnicas para caracterizar los pulsos láser ultracortos. Estas técnicas hacen uso de la autocorrelación o la correlación de las señales. Las propiedades espectrales de las funciones cuasigaussianas fueron aprovechadas para aproximar a segundo orden los coeficiente de las funciones de Hermite-Gauss que describen pulsos casi perfectos. También se discuten otras técnicas: Frequency-Resolved Optical-Gating (FROG), que muestra la dependencia temporal de la intensidad y fase del pulso láser; el sonograma del pulso producido por un detector de dos fotones, que es sensible y robusto. SPIDER que mide la interferencia entre dos pulsos separados en el tiempo indéticos excepto en su frecuencia central. Finalmente, las propiedades de las funciones ambigüedad y de Wigner se usaron para la caracterización de pulsos ultracortos.

Palabras clave: Pulsos Ultracortos, Autocorrelación, Correlación, Funciones de Hermite-Gauss, FROG, SPIDER, Función Ambigüedad, Función de Wigner.

\section{REFERENCIAS Y ENLACES / REFERENCES AND LINKS}

[1]. W. Kaiser, Edt., Generation of Ultrashort Optical Pulses: Generation and Applications, Springer-Verlag, Berlin (1993).

[2]. K. W. DeLong, R. Trebino, J. Hunter, W. E. White, "Frequency-resolved optical gating with the use of second-harmonic generation", J. Opt. Soc. Am. B 11, 2206-2215 (1994). DOI

[3]. R. Ortega-Martínez, C. J. Roman, D. Kouznetsov, "On the second Order characterization of ultrashort pulses", J. Mod. Opt. 46, 2069-2077 (1999).

[4]. I. G. Cormack, W. Sibbett, R. Ortega-Martínez, D. T. Reid, "Ultrashort pulse characterization using a scanning Fabry-Pérot étalon enabling rapid adquisition and retrieval of a sonogram at rates up to 1.52 Hz", Rev. Sci. Instrum. 72, 4071-4079 (2001). DOI 
[5]. D. T. Reid, J. Garduño-Mejía, "General ultrafast pulse measurement using the cross-correlation singleshot sonogram technique", Opt. Lett. 29, 644-646 (2004). DOI

[6]. D. T. Reid, "Algorithm for complete and rapid retrieval of ultrashort pulse amplitude and phase from a sonogram", IEEE J. Quantum Electron. 35, 1584-1589 (1999). DOI

[7]. C. Iaconis, I. A. Walmsley, "Spectral phase interferometry for direct electric-field reconstruction of ultrashort optical pulses”, Opt. Lett. 23, 792-794 (1998). DOI

[8]. T. M. Shuman, M. E. Anderson, J. Bromage, C. Iaconis, L. Waxer, I. A. Walmsley, "Real-time SPIDER: Ultrashort pulse characterization at $20 \mathrm{~Hz}$ ", Opt. Express 5, 134-143 (1999). DOI

[9]. H. Bartelt, K. H. Brenner, "The Wigner distribution function: An alternate signal representation in Optics", Israel J. Tech. 18, 260-262 (1980).

[10].S. Mukamel, C. Ciordas-Ciurdariu, V. Khidekel, "Wigner spectrograms for femtosecond pulse-shaped heterodyne and autocorrelation measurements", IEEE J. Quantum Electron. 32, 1278-1288 (1996). $\underline{\text { DOI }}$

[11].C. Dorrer, F. Salin, "Characterization of spectral phase modulation by classical and polarization spectral interferometry”, J. Opt. Soc. Am. B 15, 2331-2337 (1998). DOI

[12]. L. Lepetit, G. Cheriaux, M. Joffre, "Linear techniques of phase measurement by femtosecond spectral interferometry for applications in spectroscopy", J. Opt. Soc. Am. B 12, 2467-2474 (1995). DOI

[13].G. Castro, J. Garduño, M. Rosete, C. J. Román, R. Ortega, "High speed femtosecond pulse characterization based on spectral interferometry", Opt. Pura Apl. 46, 1-6 (2013). DOI

[14]. E. Goulielmakis, G. Nersisyan, N. A. Papadogiannis, D. Charalambidis, G. D. Tsakiris, K. Witte, "A dispersionless Michelson interferometer for the characterization of attosecond pulses", Appl. Phys. B Lasers 0. 74, 197-206 (2002). DOI

\section{Introduction}

The last forty years have brought the optical technology of ultrashort pulse generation on picoseconds $\left(10^{-12} \mathrm{~s}\right)$, femtosecond $\left(10^{-15} \mathrm{~s}\right)$ and attosecond $\left(10^{-18} \mathrm{~s}\right)$ over a wide range of the electromagnetic spectrum. Novel ultrafast optical modulation techniques used for selfmode-locked Ti:Sapphire and solid state lasers have allowed powerful ultrafast pulse generation with high stability, reliability and reproducibility. The development of high repetition rate ultrashort pulsed laser sources have been used in a great variety of fields in science and technology. Electronic detectors are not capable to resolve a femtosecond pulse and then alternative techniques must be applied [1]. The autocorrelation (or cross-correlation) techniques combined with the signal pulse spectrum clearly characterize the intensity and phase of the laser pulse. Roughly, the optical pulse can be characterized by its spectral width $\Delta \omega$ and by its time duration $\Delta \tau$. An almost perfect pulse means an almost Gaussian profile without chirp $(\Delta \tau \Delta \omega \approx 1 / 2)$. The product $\Delta \tau \Delta \omega$ can be considered as a measurement of the perfection of the pulse. We assume that a perfect pulse has no internal structure is as low as possible. This means that the squared modulus of the electric field $E(t)$ and its Fouriertransform $\tilde{E}(\omega)$ have an almost-Gaussian profile, such that $(\Delta \tau)^{2}(\Delta \omega)^{2} \sim 1 / 4$ should be accomplished. The Ti:Sapphire laser is the most common source of ultrashort laser pulses which are time measured with an autocorrelator (Fig. 1). The incoming pulses at $\lambda_{0}=800 \mathrm{~nm}$ are split by a pellicle beam splitter into two replicas, one of them travels a variable-delay line while the other goes through a fixed-delay line. A focus mirror combines both replicas on a KDP nonlinear crystal producing a second harmonic signal of fundamental pulses $\left(\lambda_{2}=400 \mathrm{~nm}\right)$. This signal is captured by a photomultiplier tube and displayed on the digital oscilloscope of large bandwidth. A rotating mirror stage supplies the variable-delay line. A corner-cube retro-reflector mirror on a sliding stage gives the fixed-delay path (Fig. 1).

\section{The techniques}

The characterization of an optical pulse by its intensity profile and temporal phase or intensity of its spectrum and spectral phase is also interesting due to the analogy with the reconstruction of the transverse structure of a 


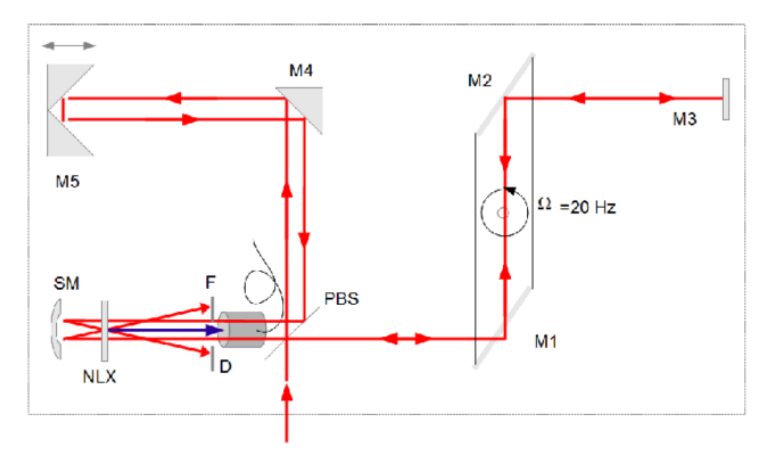

Fig. 1. Autocorrelator Femtochrome ${ }^{\mathrm{TM}}$ FR-XL130.

monochromatic beam by its intensity detected in the image and Fourier planes. Distortions of the perfect Gaussian pulse are properly described by Hermite-Gauss functions which are self-Fourier transformed, and which give information about the class of these distortions. Several techniques have been used in the Laboratory to characterized laser pulses: autocorrelation, frequency-resolved optical gating, sonogram, spectral-phase interferometry and theoretically the Ambiguity-Wigner functions. The experimental measurements have been made for $100 \mathrm{fs}$, with a spectral width of $\sim 7 \mathrm{~nm}$ at nearinfrared and visible [2,3]. A brief discussion on SPIDER also is included, in order to give a more completed survey of the more common techniques used to measure ultrashort pulses.

\subsection{Second order approximation}

Let the electric field of the laser pulse to be $E(t)$ and its intensity profile $|E(t)|^{2}$ and the spectral intensity profile $|\tilde{E}(\omega)|^{2}$, and $\left|E_{0}(t)\right|^{2}$ and $\left|\tilde{E}_{0}(\omega)\right|^{2}$ the corresponding quantities to another pulse $E_{0}$. The intensity is measure with some error, so the difference can be written by a defined error-bar functions:

$$
\begin{gathered}
U=\int_{-\infty}^{+\infty}\left(|E(t)|^{2}-\left|E_{0}(t)\right|^{2}\right)^{2} d t \\
W=\int_{-\infty}^{+\infty}\left(|\tilde{E}(\omega)|^{2}-\left|\tilde{E}_{0}(\omega)\right|^{2}\right)^{2} d \omega .
\end{gathered}
$$

Both functions are in principle measurable. We usually have autocorrelation in Eq. (1) instead of the intensity for ultrashort pulses. It has been shown that, from the modulus of the function and the modulus of its Fourier- transform, the function can be effectively reconstructed [3], at least if the function has a finite support. This means that a segment $t_{1}<t<t_{2}$ exists such a function $E(t)=0$ if $t \leq t_{1}$ or $t \geq t_{2}$. So, we could expect that the difference between $E$ and $E_{0}$ could cause a significant increase of the error-bar functions $U$ and $W$. For the Hermite-Gauss functions, we have the following theorem:

$$
\mathcal{F}\left\{H_{n}(t) e^{-\frac{t^{2}}{2}}, t ; \omega\right\}=i^{n} H_{n}(\omega) e^{-\frac{\omega^{2}}{2}} .
$$

This is clear if we treat $H_{n}(t) e^{-t^{2} / 2}$ as an eigenfunction of the Hamiltonian of the quantum harmonic oscillator. A complete proof of this theorem which uses only the properties of the Hermite polynomials and no quantum analogies is shown in [3]. Therefore, distortions will be hard to define at quadratic order perturbations with respect to $\varepsilon$. At first approximation, distortions that do not change $U+W$ can be expressed as:

$$
\delta E(t)=\sum_{n=2}^{\infty} i \varepsilon_{n} \widetilde{H}_{2 n}(t) e^{-\frac{t^{2}}{2}},
$$

where the $\varepsilon_{n}$ are small amounts and $\widetilde{H}(t)=H(t) / \sqrt{2^{n} \sqrt{\pi} n}$ !. In the linearized approximation, $E(t)$ and $E(t)+\delta E(t)$ have the same square of the modulus and the same square of the Fourier transform. Now, we show the distortions of the pulse in the frequency domain but not in the time domain, which can be described by Eq. (5). In the dimensionless coordinates $t \rightarrow\left(t-t_{0}\right) / \Delta \tau, \omega \rightarrow\left(\omega-\omega_{0}\right) / \Delta \omega$, we simulate the characterization with quadratic detectors of the self-Fourier Gaussian $u_{0}(t)=\exp \left(-t^{2} / 2\right)$. In the simplest case, we disturb this perfect Gaussian $u_{0}$ with a small amount of the normalized Hermite-Gaussian function, so the pulse $u$ has the form:

$$
u(t)=\left[1+\alpha \widetilde{H}_{n}(t)\right] e^{-\frac{t^{2}}{2}},
$$

with $\alpha=i \varepsilon$. Some of our results are illustrated in Figs. 2 to 5 with Hermite-Gaussian functions of third and fourth order and $\alpha=1 / 8$.

Examples are given for the spectra of the pulse in the time and frequency domains. Figure 2 is the square of the modulus of $u_{0}(t)=$ $\exp \left(-t^{2} / 2\right)$ and $u(t)=\left|1+i / 8 \widetilde{H}_{3}(t)\right| \exp \left(-t^{2}\right)$. 


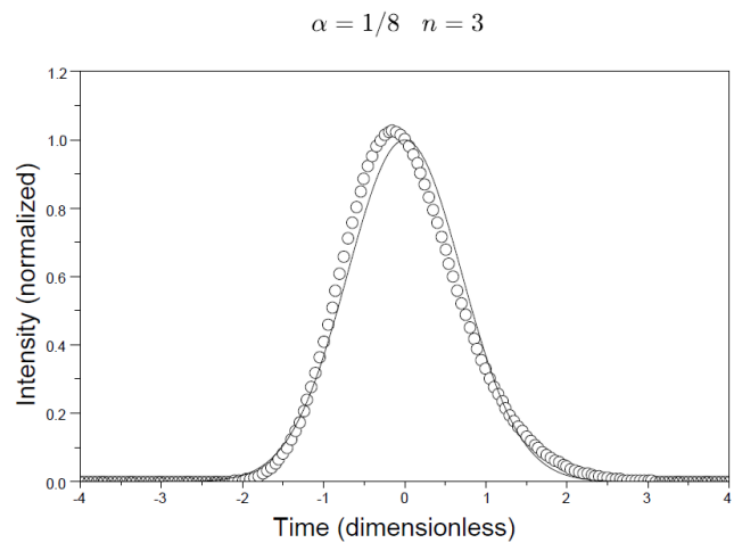

Fig. 2: Square modulus $\left|u_{0}(t)\right|^{2}$ (continuous line) and $|u(t)|^{2}=\left|1+1 / 8 \widetilde{H}_{3}(t)\right| \exp \left(-t^{2}\right)(0)$.

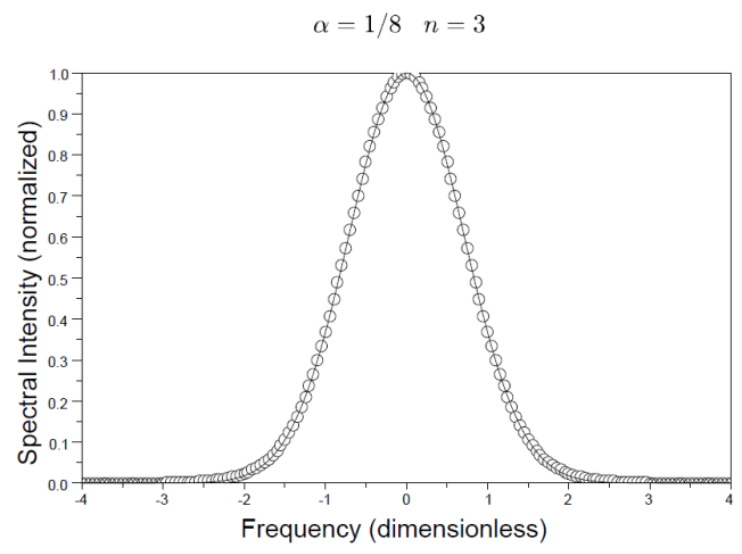

Fig. 3: Square modulus $\left|u_{0}(\omega)\right|^{2}$ (continuous line) and $|u(\omega)|^{2}=\left|1+1 / 8 \widetilde{H}_{3}(\omega)\right| \exp \left(-\omega^{2}\right)(\circ)$.

Figure 3 is the square of the modulus of $\tilde{u}_{0}(\omega)=\exp \left(-\omega^{2} / 2\right) \quad$ and $\quad u(\omega)=\mid 1+$ $i / 8 \widetilde{H}_{3}(\omega) \mid \exp \left(-\omega^{2}\right)[3]$.

\subsection{Frequency-resolved optical gating}

FROG $^{\mathrm{TM}}$ is a technique for measuring the timedependent (or, equivalently, frequencydependent) intensity and phase of an ultrashort laser pulse. It is rigorous, general, and simple. It is a powerful diagnostic device for deriving both the full intensity and phase of arbitrary-shaped ultrashort laser pulse. This technique combines a visual spectrogram with an iterative algorithm to extract the quantitative data. It consists of a simple apparatus that generates a spectrogram of the laser pulse, also known as a "FROG trace", along with an algorithm that retrieves the timedependent intensity and phase of the pulse that generated the trace. The technique does not require a priori assumption about the nature of

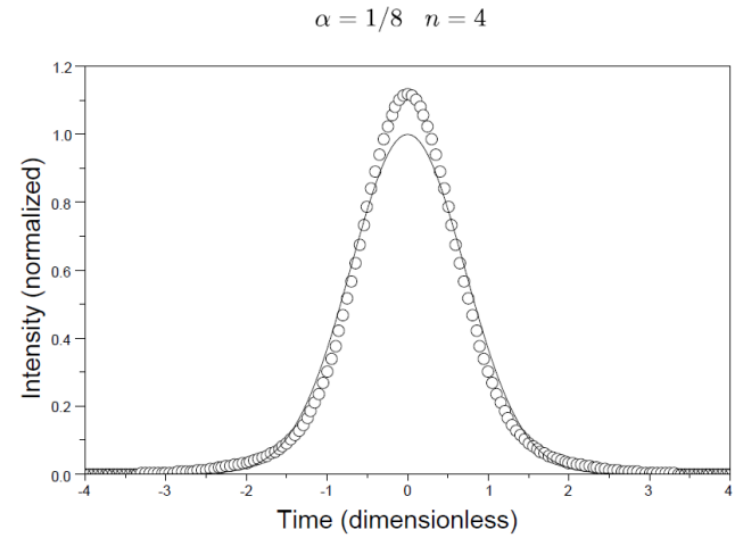

Fig. 3: Square modulus $\left|u_{0}(t)\right|^{2}$ (continuous line) and $|u(t)|^{2}=\left|1+1 / 8 \widetilde{H}_{4}(t)\right| \exp \left(-t^{2}\right)(0)$.

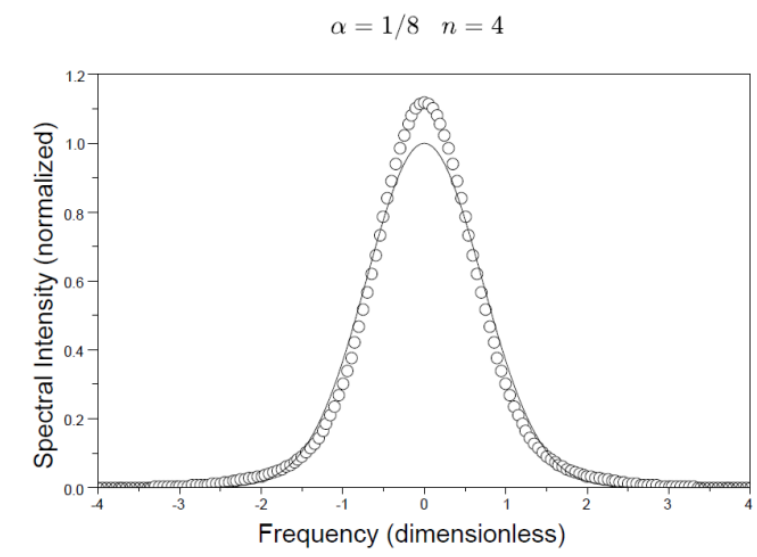

Fig. 5: Square modulus $\left|u_{0}(\omega)\right|^{2}$ (continuous line) and $|u(\omega)|^{2}=\left|1+1 / 8 \widetilde{H}_{4}(\omega)\right| \exp \left(-\omega^{2}\right)(\circ)$.

the pulse, it depends only on the nonlinear medium response, which is known [2]. FROG is in essence an autocorrelator or cross-correlator, but rather than measuring the signal's energy vs delay, the technique involves measuring the signal pulse's spectrum vs delay. Specifically the pulse to be measured is split into two replicas. These two replicas are crossed in a nonlinear optical medium to create a signal field, as in autocorrelation. The signal is then spectrally resolved. Any autocorrelator or cross-correlator beam geometry may be used, with a polarization-gate geometry (PG-FROG) or second harmonic-generation (SHG-FROG), yields the most intuitive traces. In the SHG-FROG geometry, the non-collinear autocorrelation signal generated in a frequency-doubling crystal is the nonlinear signal. The two electric field replicas of the pulse are usually called probe $E(t)$ and the variable delay gate $g(t-\tau)=$ $E(t-\tau)$. The gate beam overlaps with the help of 
a lens the probe beam in the nonlinear optical medium (typically KDP and $\beta$-borate). In the case of SHG-FROG the noncollinear autocorrelation signal generated in a frequencydoubling crystal is the nonlinear signal having the form:

$$
E_{s i g}(t, \tau)=E(t) E(t-\tau),
$$

where $E(t)$ is the complex amplitude of the pulse to be measured. This signal field is the frequency resolved by a spectrometer, that is, its spectrum is measured. The spectrum of the signal field is measured for all relevant values of the delay time between the two beams to yield the trace:

$$
I_{F R O G}(\omega, \tau)=\left|\int_{-\infty}^{+\infty} E_{s i g}(t, \tau) e^{-i \omega t} d t\right|^{2},
$$

where $\omega$ is the frequency and $\tau$ is the time delay. The FROG trace is a spectrogram, that illustrates the pulse frequency delay-time, analogous to a musical score, illustrating the pulse frequency vs time-series analysis. It can also be thought of as the output of a graphic equalizer for ultrashort laser pulses. A experimental layout is given in Fig. 6. The FROG trace is a positive real-valued function of two variables: the frequency and the time delay between the two pulses. This experimentally determined FROG trace is then used as an input to a numerical algorithm, which determines the full complex electric field, i.e. both the intensity and the phase. The spectrogram is obtained by taking a series of spectra of the gated pulsed as a function of the gate delay. The spectra may be gathered simultaneously (single shot) or sequentially (scanning), depending on the intensity of the pulse and the nature of the gating technique used. The measured spectrogram, independent of the electric-field retrieval, can be used immediately as a pulse diagnostic. This is due to the fact that different distortions of the pulse in both intensity and phase produce spectrograms. To better understand how spectrograms work, and in particular, how FROG works, imagine one replica of a positively chirped pulse gating the other. The gate pulse slices out a temporal chunk is in the positive trailing edge of the pulse (for a Gaussian-intensity pulse, the temporal chunk occurs at $2 / 3$ of the delay). As a result, for the

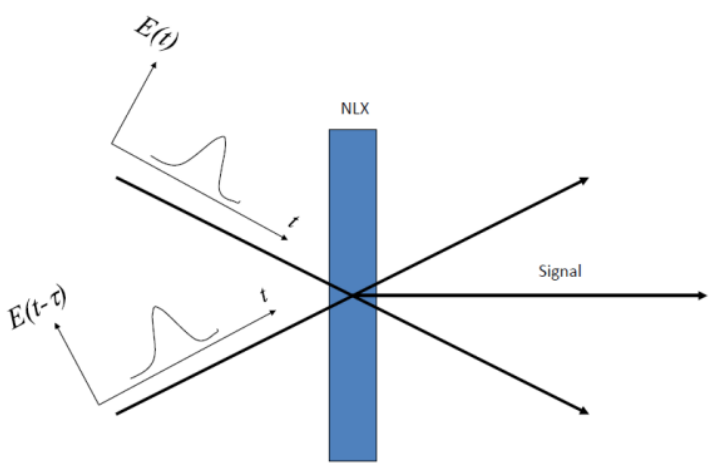

Fig. 6: FROG scheme.

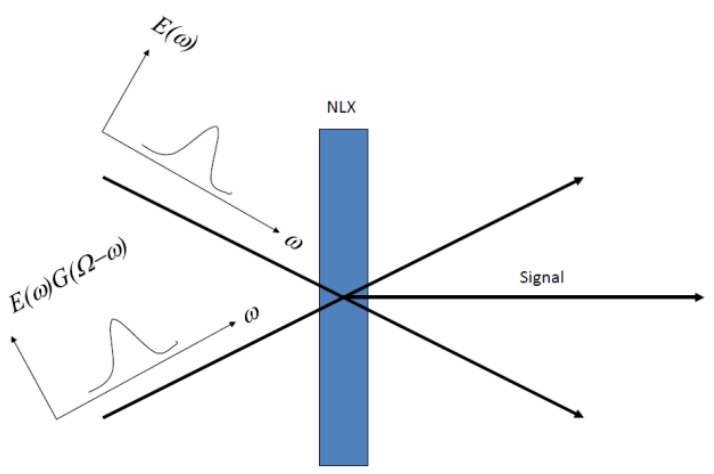

Fig. 7: Sonogram scheme.

above positively chirped pulse, it has a color and intensity. As the gate is scanned in time, the chunk's intensity and color will be different, depending on the intensity and color of the pulse. Thus, it can be seen that measuring the spectrum of each of the chunks will fairly well determine the pulse intensity and color (or frequency or phase) vs time.

\subsection{Sonogram}

The sonogram is generated by filtering the signal in the frequency domain, and by recording the resultant output as a function of time (Fig. 7). The group delay of each frequency component using a frequency-filter $G(\omega)$ function followed by a time-resolved measurement of its intensity:

$$
\begin{aligned}
I_{\text {SONO }}(\tau, \Omega) & = \\
& =\left|\int_{-\infty}^{+\infty} E(\omega) G(\omega-\Omega) e^{-i \omega t} d \omega\right|^{2} .
\end{aligned}
$$

Experimentally, the sonogram can be recorded by splitting a pulse into two replicas, frequency filtering one of these using a dispersion-free 
spectrometer, then cross-correlating the pulses using a second order nonlinearity. Unlike SHGFROG a sonogram is sensitive to the sign of the chirp of the pulse, making it a potentially superior measurement to SHGFROG. A further advantage of sonogram measurement is that, unlike SHG-FROG, it can be recorded using a two-photon absorption detection with a Al-GaAs LED or a GaAsP photodiode, to provide the nonlinearity, therefore allowing characterization of extremely short pulses without introducing systematic errors arising from the limited phasematching bandwidth of a frequency-doubling crystal $[4,5]$. D. T. Reid reported and algorithm for the retrieval of the main ultrashort pulse parameter from a sonogram [6]

\subsection{SPIDER}

SPIDER uses spectral shearing interferometry to retrieve the spectral phase of the incident pulse. This form of interferometry measures the interference between two pulses separated in time which are identical except for their central frequencies. This pulse pair is an inteferogram signal on the form:

$$
\begin{aligned}
S(\omega)=I(\omega) & +I(\omega+\Omega)+ \\
+ & 2 \sqrt{I(\omega) I(\omega+\Omega)} \times \\
& \times \cos \varphi[(\omega+\Omega)-\varphi(\omega)+\omega \tau],
\end{aligned}
$$

where $\Omega$ is the spectral shear (the difference between the central frequencies of the individual pulses in the pair), $\tau$ is the time delay between the pulses, $I(\omega)$ is the pulse spectrum and $\varphi(\omega)$ is the spectral phase of the pulse. The time delay is introduced to facilitate phase recovery and is equivalent to the introduction of tilt fringes in spatial interferometry. A complete specification of the electric field of the pulse in the frequency domain is given by the pair of functions $\sqrt{I(\omega)}$ and $\varphi(\omega)$. The former is easily measured with this apparatus and the latter can be extracted from the SPIDER interferogram [7].

The SPIDER technique consists on generates a spectral interferogram between a pulse and its spectrally sheared and delayed pulse. In order to produce a shear of the required magnitude (typically a significant portion of the pulse spectra width, or for femtosecond pulses several $\mathrm{THz}$ ), a nonlinear process must be used. Both upconversion and down-conversion can be used for this purpose, depending on the central wavelength of the pulses being characterize. By mixing two properly beams in a nonlinear crystal, the resulting sum or difference frequency radiation consists of a spectrally sheared pulse pair. One of the beams incident on the crystal has a pair of identical pulses (replicas of the pulses being characterized), separated completely by a time delay $\tau$. The other beam contains a highly chirped pulsed. The chirp on this pulse should be large enough to satisfy two conditions. First, the duration of the chirp pulse should be much longer than the time delayed $\tau$. Second, during each of the replica pulses, the chirped pulse is essentially monochromatic. This ensures that when these two beams mix in the crystal, each pulse in the pair mixes with different frequency in the chirped pulse. The pulses emerging from the crystal, therefore, are each shifted to a different frequency, i.e. they are spectrally sheared. The magnitude of the spectral shear generated in this manner is a function of two parameters: the separation between pulses in the pair and the amount of chirp on the stretched pulse. For pulses stretched using a typical grating stretcher in which second order dispersion dominates, the exact value of the shear is equal to $\Omega=-\tau / 2 \varphi_{2}$ where $\tau$ is the separation between the pulses and $\varphi_{2}$ is the second-order dispersion presented on the chirped beam. In this case, the value of ' 2 is determined from the relations stated in the [7]. Once the interferogram signal is resolved, it is sampled with a detector at the output of a spectrometer [8].

\section{The ambiguity and Wigner functions}

The ambiguity function $A$ of the function $E(t)$ in the time-domain is defined by:

$$
\begin{aligned}
A(\tau, v) & = \\
& =\int_{-\infty}^{+\infty} E\left(t+\frac{\tau}{2}\right) E^{*}\left(t-\frac{\tau}{2}\right) e^{i 2 \pi v t} d t,
\end{aligned}
$$

and the Wigner function on the time-domain is defined by: 


$$
\begin{aligned}
W(\tau, v) & = \\
& =\int_{-\infty}^{+\infty} E\left(\tau+\frac{t}{2}\right) E^{*}\left(\tau-\frac{t}{2}\right) e^{i 2 \pi v t} d t
\end{aligned}
$$

or, equivalently in the frequency domain:

$$
\begin{aligned}
& W(\tau, v)= \\
& \quad=\int_{-\infty}^{+\infty} \tilde{E}\left(v+\frac{v^{\prime}}{2}\right) \tilde{E}^{*}\left(v-\frac{v^{\prime}}{2}\right) e^{i 2 \pi v t} d v^{\prime},
\end{aligned}
$$

where $E(t)$ and $\tilde{E}(v)$ form a pair of Fourier transforms, i.e. $\tilde{E}(v)=\int_{-\infty}^{+\infty} E(t) e^{-i 2 \pi v t} d t$.

The ambiguity and Wigner functions are related by a 2-D Fourier transform as given by the following expression:

$W(\tau, v)=\int_{-\infty}^{+\infty} \int_{-\infty} A\left(\tau^{\prime}, v^{\prime}\right) e^{i 2 \pi\left(\tau v^{\prime}+\tau^{\prime} v\right)} d \tau^{\prime} d v^{\prime}$,

where the primed are dummy variables.

These functions represents a Fourier pair which can be use for the ultrafast laser pulse characterization. It can be demonstrated the pulse intensity and power spectrum retrieval for different amounts of linear and second order chirp $[9,10]$. For the full characterizations spectral interferometry technique for phase retrieval can be applied. This method is very sensitive since does depends on a linear response with the only condition that we must provide a reference pulse. The technique consists on resolve, in the frequency domain, the interferogram generated by the pulse to be characterized and its delayed (fixed) reference pulse in a spectrometer. Actual spectral phase information is retrieved by a Fourier Transform, side band filtering and phase unwrapping process. To complete the computation, actual spectral phase of the reference pulse is subtracted [11]. For instance, if the pulse reference is bandwidth limited (BL), spectral phase calculation of a shaped pulse is then a straight forward process since the algorithm result will be directly the unknown spectral phase [12].

The applications of WF to signal analysis in the space or time domain have been done in the last thirty years and a new interpretation may be fruitful. In the time domain, the development has followed a different route: "instantaneous frequency" analyzers of analogue acoustic or radio signals were produce with electronic resonators more than a century ago, and something like the Wigner function was developed even earlier for the representation of music notes on a pentagram $[2,9]$.

When light is used to produce time signals through intensity modulation of an ultrashort pulse of a laser beam, the corresponding Wigner function is essentially that of quantum mechanics, with the reduced wavelength $\lambda / 2$ taking place of Planck's constant $\hbar$. Such beams are monochromatic, and time modulation spreads their color negligibly. For light pulses of femtosecond and attosecond pulse duration, where the "quasi"-monochromatic approximation no longer holds, the output contains a wide color spectrum. Here we present preliminary theoretical simulation results using the demodulated ambiguity and Wigner functions (that will be reported elsewhere) of pulses with linear and quadratic chirps, for $\tau_{p}=10$ as at $\lambda=0.5 \mathrm{~nm}$ (Figs. 8, 10, 12 and 14).

The intensity is given by:

$$
I(\tau)=\int_{-\infty}^{+\infty} W(\tau, v) d v
$$

and the power spectrum:

$$
S(v)=\int_{-\infty}^{+\infty} W(\tau, v) d \tau
$$

See Figs. 9, 11, 13 and 15. The quadratic and cubic phase retrievals are shown in Figs. 16 and 17.

The spectral interferometry technique is used for the retrieval of the phases in previous cases $[12,13]$.

\subsection{Comments on attoseconds measurements techniques}

The temporal characterization of ultrashort pulses requires the use of at least second-order autocorrelation and hence of a non-linear detector with sufficient bandwidth and sensitivity. In the case of femtosecond pulses with frequencies in the visible spectral region, an amplitude-splitting Michelson interferometer in conjunction with a second-harmonic crystal is 


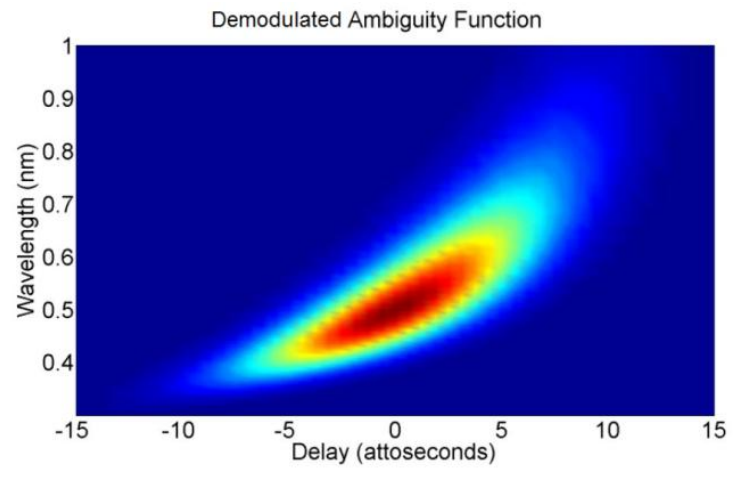

Fig. 8: Demodulated ambiguity function for a $\tau_{p}=10$ as at $\lambda=0.5 \mathrm{~nm}$ with linear chirp.

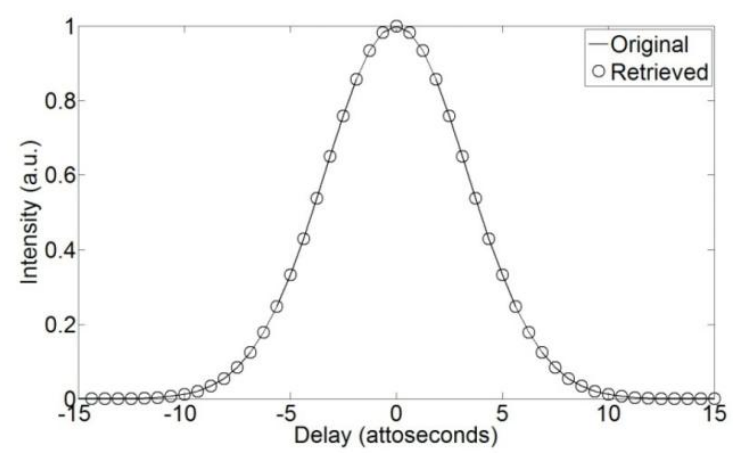

Fig. 9: Pulse intensity profile for a $\tau_{p}=10$ as at $\lambda=0.5 \mathrm{~nm}$ with linear chirp.

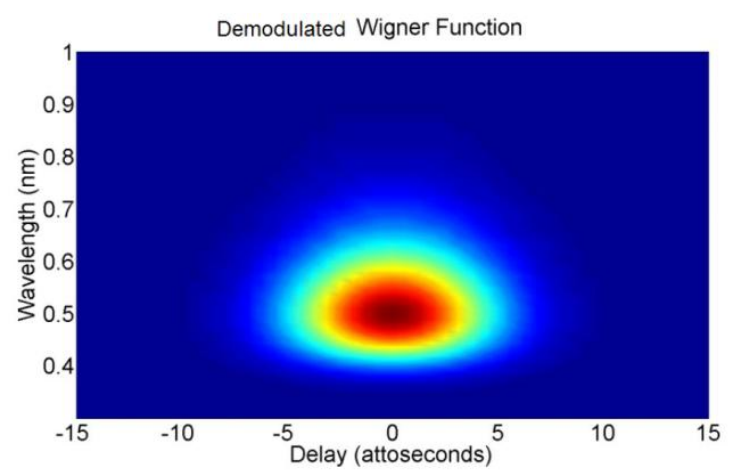

Fig. 10: Demodulated Wigner function for a $\tau_{p}=10$ as at $\lambda=0.5 \mathrm{~nm}$ with linear chirp.

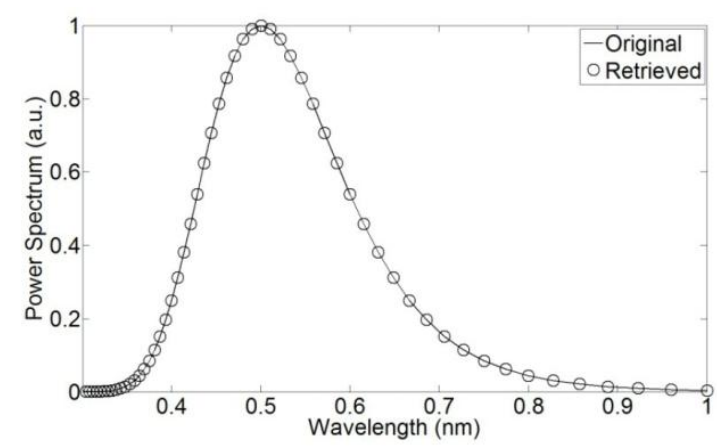

Fig. 11: Power spectrum profile for a $\tau_{p}=10$ as at $\lambda=0.5 \mathrm{~nm}$ pulse with linear chirp.

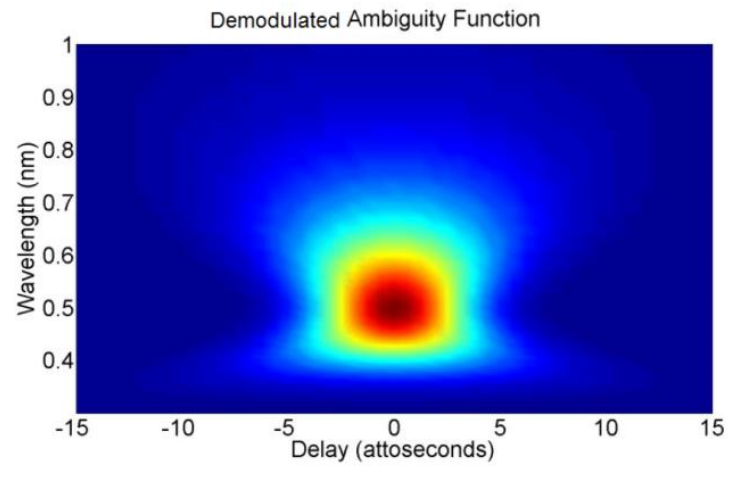

Fig. 12: Demodulated ambiguity function of a $\tau_{p}=10$ as at $\lambda=0.5 \mathrm{~nm}$ pulse with quadratic chirp.

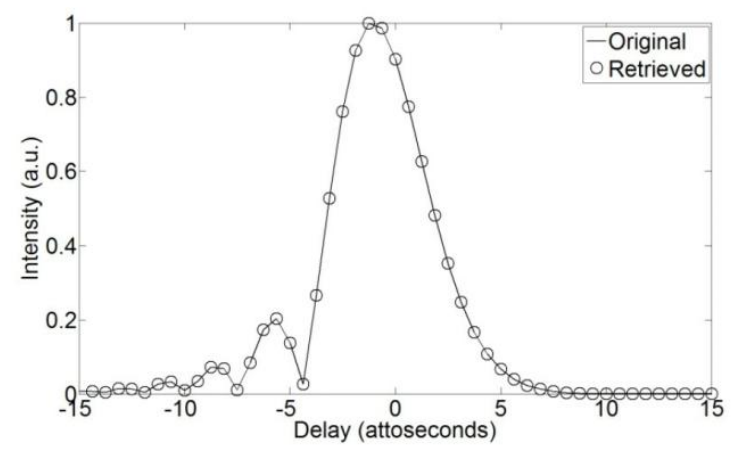

Fig. 13: Pulse intensity profile of a $\tau_{p}=10$ as at $\lambda=0.5 \mathrm{~nm}$ pulse with quadratic chirp.

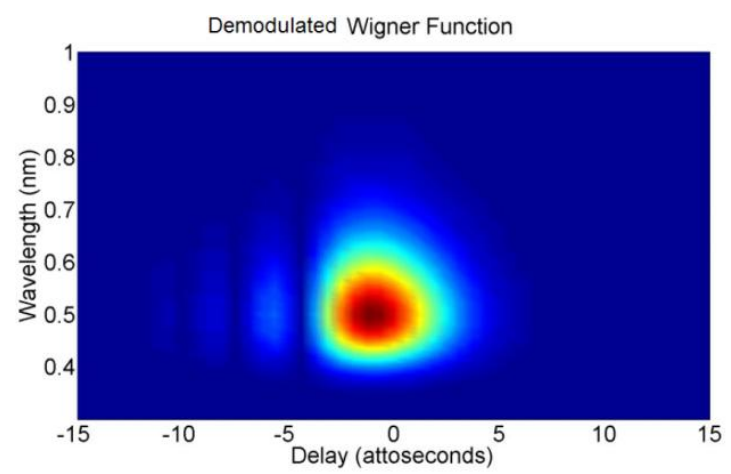

Fig. 14: Demodulated Wigner function of a $\tau_{p}=10$ as at $\lambda=0.5 \mathrm{~nm}$ pulse with quadratic chirp.

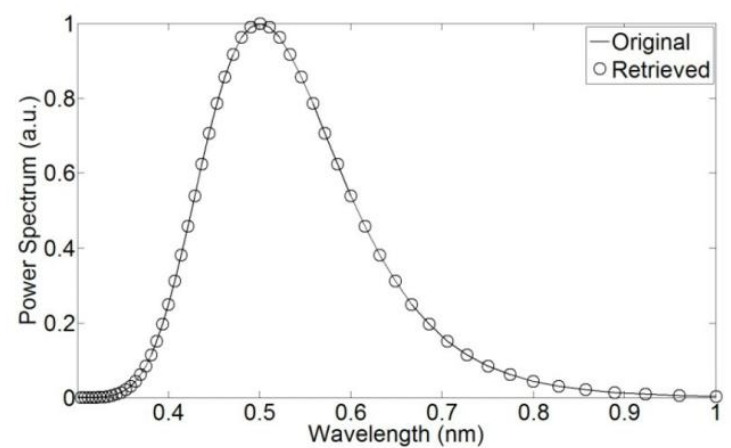

Fig. 15: Power spectrum profile of a $\tau_{p}=10$ as at $\lambda=0.5 \mathrm{~nm}$ pulse with quadratic chirp. 


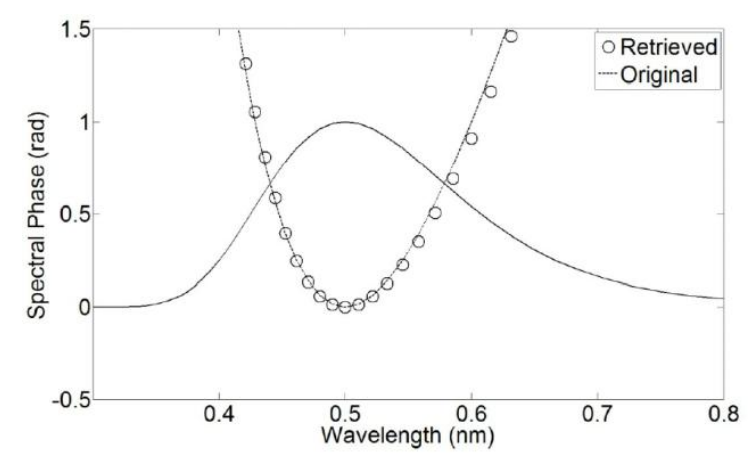

Fig. 16: Quadratic phase retrieval for the first case using spectral phase interferometry.

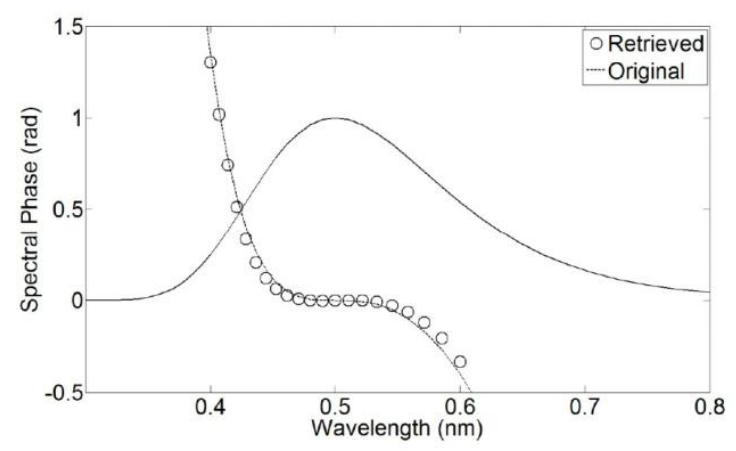

Fig. 17: Cubic phase retrieval for the second case using spectral phase interferometry.

commonly used. The conventional Michelson interferometer for the characterization of femtosecond pulses in autocorrelation techniques uses a pellicle beam splitter. The extension of the Michelson interferometer for operation in the XUV spectral range uses a freestanding transmission grating as a beam splitter [14].

\section{Conclusions}

In this contribution we present numerical and experimental results for several techniques for the characterization of ultrashort laser pulses. We also present the class of perturbation that cannot be detected in intensity and spectrum intensity of Gaussian pulses. A suitable option for ultrashort pulse characterization is spectral interferometry, a method that depends on a linear response of the phase retrieval procedure. Promising new experimental results are expected to be reported elsewhere for ultrashort pulses less than 50 fs in near infrared and theoretical simulations for attoseconds.

\section{Acknowledgements}

The author thanks to J. Garduño-Mejía and C. J. Román-Moreno for the assistance in preparation this work. He also thanks the sponsorship by BID-UNAM \#98-B3-C-DGP-L0034-1077, DGAPAUNAM PAPIIT projects: IN104597, IN108900 and CONACyT-Mexico projects: 4480-A9409, 34921-E. R. Ortega (\#6095) is Member of the Sistema Nacional de Investigadores. 